0

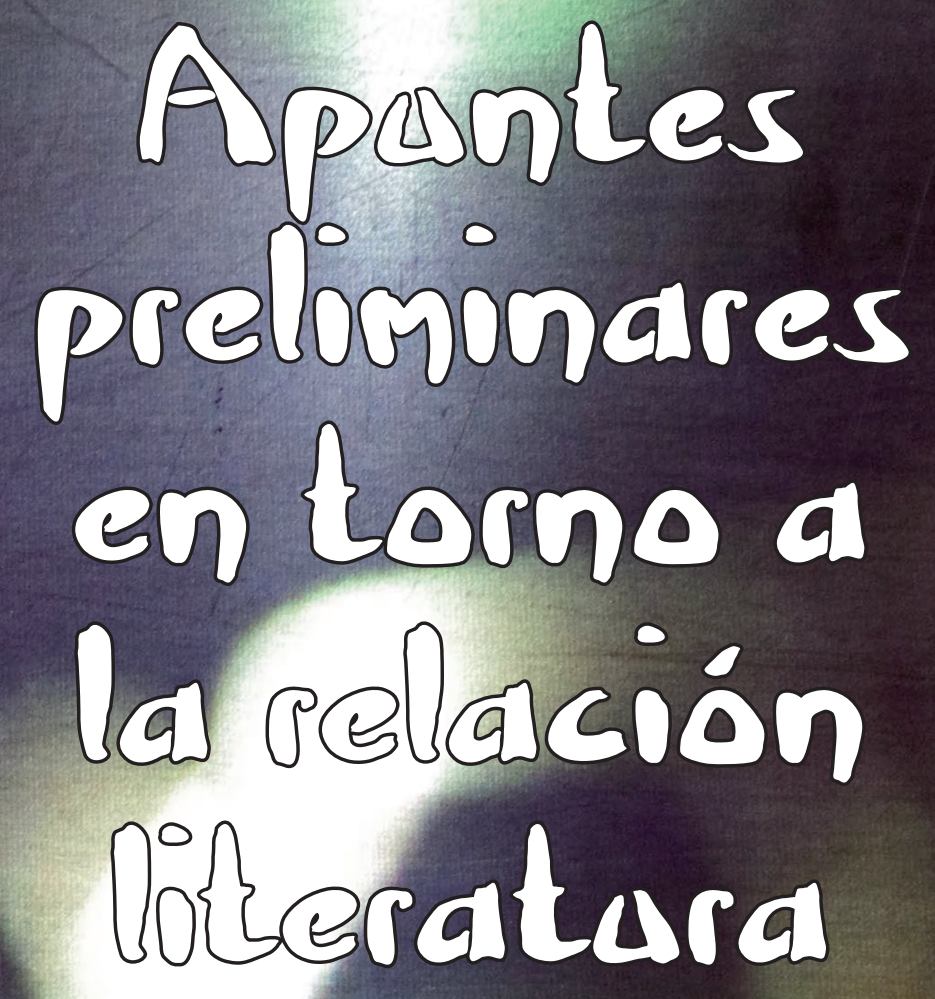

faroúástica y comlexto

3 + Nadia Juliana Bazán 


\section{Apuntes preliminares en torno a la relación literatura fantástica y contexto ${ }^{1}$}

\section{Resumen}

La literatura fantástica es entendida como la transgresión a un orden establecido y, a su vez, es uno de los géneros que más revela los contextos sociales y culturales que la originan; en este artículo se referencia el ejemplo de los inicios de la literatura fantástica en el Cono Sur (Argentina y Uruguay) en dos autores representativos: Leopoldo Lugones y Horacio Quiroga. En estos dos autores la literatura fantástica es utilizada como herramienta para remover los cimientos de la lógica tradicional europea, proponiendo una transgresión del orden establecido que rige su sociedad e insistiendo en "otras formas" de entender la propia realidad (es decir: el contexto). Lo fantástico en Lugones y Quiroga (y más adelante en Borges y Cortázar) quebranta la realidad real al estructurar una transgresión de la racionalidad y la lógica europea producto de la modernidad.

Palabras clave: Realidad real, literatura fantástica, suceso fantástico, Leopoldo Lugones y Horacio Quiroga.

\section{Preliminary notations concerning the relation ship between fantastic literature and context}

Abstract: Fantastic literature is commonly understood as the transgression of an established order and, in its turn, it is also one of the literary genres that better underline social and cultural backgrounds. This paper recalls the beginnings of Fantastic Literature in the Southern Cone (Argentina and Uruguay), taking two representative authors as a particular reference: they are Leopoldo Lugones and Horacio Quiroga. Fantastic literature is used by them as an instrument capable of shaking the very foundations of traditional European logics, therefore transgressing the established order that controls their societies and underlining "other ways" of understanding their very reality (the context). In Lugones and Quiroga (as latter in Borges and Cortázar), the fantastic undermines actual reality by means of structuring the transgression of European logics and rationality, which are a result of modernity.

Keywords: Actual reality, fantastic literature, fantastic event, Leopoldo Lugones, Horacio Quiroga.

\section{Apontamentos preliminares em volta a relação literatura fantástica e contexto}

Resumo: A literatura fantástica e percebida como a transgressão a uma ordem estabelecida e ao mesmo tempo, e um dos gêneros que mais destacam os contextos sociais e culturais; neste artigo se faz referencia ao exemplo dos inícios da literatura fantástica no Cone Sul (Argentina e Uruguai) em dois autores representativos: Leopoldo Lugones e Horacio Quiroga. A literatura fantástica então, e utilizada como ferramenta para remover os cimentos da lógica tradicional europeia, propondo uma transgressão da ordem estabelecida que reja sua sociedade, e insistindo em "outras formas" de entender a própria realidade (ou seja: o contexto). O fantástico em Lugones e Quiroga (e mais para frente em Borges e Cortázar) rompe a realidade real estruturando uma transgressão da racionalidade e da lógica europeia produto da modernidade.

Palavras chave: realidade real, literatura fantástica, acontecimento fantástico, Leopoldo Lugones e Horacio Quiroga.

1 El lector encontrará aquí fragmentos de mi tesis de grado como antropóloga con opción en teoría musical, denominada: ¿`No es real lo fantástico? La literatura fantástica en el Cono Sur en relación con su contexto social y cultural” (Bazán, 2002). 
¿Puede la literatura fantástica permitirnos pensar los contextos históricos que la hicieron posible? ¿Cómo hacerlo? ¿Con qué metodologías y análisis? ¿Cómo Leopoldo Lugones y Horacio Quiroga nos remiten al contexto del Cono Sur latinoamericano a través de su literatura fantástica?

En la antología de cuentos fantásticos, recopilada por Italo Calvino se encuentra la siguiente aseveración: "El cuento fantástico es uno de los productos más característicos de la narrativa del siglo XIX y, para nosotros, uno de los más significativos, pues es el que más nos dice sobre la interioridad del individuo y de la simbología colectiva" (Calvino, 1987, p. 9). Más adelante, explica cómo "para nuestra sensibilidad de hoy", lo sobrenatural en estas narraciones está "cargado de sentido", como una "revelación de lo inconsciente, de lo reprimido, de lo olvidado, de lo alejado de nuestra atención racional".

Es a partir de esta aseveración de Italo Calvino que me decido a retomar algunos ejemplos de literatura fantástica en el Cono Sur latinoamericano, exactamente en el contexto argentino y uruguayo, a través del análisis de los cuentos de Leopoldo Lugones y Horacio Quiroga, con el fin de revisar la mencionada relación entre literatura y contexto; indagar sobre el entorno social que rodea un género tan particular (en el tipo e influencia que dicho entorno puede generar sobre este género y viceversa); como también las consecuencias que sobre una población determinada puede llegar a originar una narrativa en la cual la explicación racional y la aceptación sobrenatural conviven sin rozar con el absurdo.

\section{Discusiones en torno a la literatura fantástica}

Se puede decir que a partir de los análisis realizados por Tzvetan Todorov (1998), Luis Vax (1980), Angel Nogueira (1994) y Barella, Guerri y Carbajo (1994), que el género fantástico se sitúa entre dos géneros vecinos: lo extraño (sobrenatural explicado) y lo maravilloso (sobrenatural aceptado). Según estos autores, en lo fantástico deben coexistir los dos anteriores, es decir, debe encontrarse una vacilación entre uno y otro, en donde el lector no esté seguro si los eventos sobrenaturales del relato tienen una explicación racional o son producto de fuerzas o seres suprahumanos.

En el prólogo a la Antología de la literatura fantástica, Adolfo Bioy Casares (1967) difiere de la definición que sobre el género propone Tzvetan Todorov (1998). Mientras este último encuentra diferencias cualitativas irreconciliables entre los géneros: maravilloso, extraño y fantástico, para Bioy Casares estos forman parte de un solo género: el fantástico; y es esta la razón por la cual dentro de su antología se encuentran recopilados cuentos que, según la definición hecha por Todorov, no podrían estar contenidos en la misma.
Sin embargo, Vax (1980) avala lo planteado por Todorov, al considerar como característica esencial del género fantástico la "ambigüedad" (Todorov la denomina "vacilación") en lo referente a poder determinar si los hechos sobrenaturales narrados son reales o imaginarios, lo que para Bioy Casares (1967) no constituye necesariamente una condición sine qua non. Este último se aleja de los otros dos autores cuando asegura que parte de la "naturaleza" de un cuento fantástico es ser "un relato destinado a producir en el lector un estremecimiento particular, una exquisita angustia" (Bioy Casares, 1967, p. 15). Esto es compartido por Harry Belevan (1994). Italo Calvino (1987), en la discusión acerca de la definición de lo fantástico, encuentra, al igual que Todorov, una diferencia clara entre los géneros fantástico y maravilloso; no así el extraño el cual incluye dentro de su antología como si fuese fantástico.

Es interesante la concepción que Vax (1980) tiene acerca de lo que constituye el suspenso en estas narraciones, proponiendo que se trata de la mezcla ("ambigüedad") entre algo real y algo no real; asegura que un cuento asusta menos si el hecho sobrenatural es claramente especificado como no real; todo lo contrario ocurre en un relato donde no esté claro si lo es o no. Según Roger Caillois (citado por Barella et al., 1994), lo fantástico constituye una ruptura del orden cognoscible; se trata de tomar los elementos de la realidad, y organizarlos de manera inhabitual. De la misma manera, Angel Nogueira asegura que la literatura fantástica establece una "ruptura y apertura a la ambigüedad e incertidumbre, a la trascendencia de cuanto en lo cotidiano acontece" (Nogueira, citado por Barella et al., 1994, p. 6), pero siempre dentro de lo "racional, cotidiano y real", de manera que logra subvertir la realidad transgredirla dentro de sus propios límites. 
Harry Belevan (1976) afirma que si bien toda literatura se debate entre lo real y lo irreal, la literatura fantástica hace de este elemento "su centro explícito". De la misma opinión es Violeta Pérez Gil:

Relato fantástico presenta lo inexplicable y contradictorio de la realidad, hace intervenir lo inverosímil, de modo que el orden establecido queda en entredicho gracias a un fenómeno que se sale de sus reglas de juego, enfrentando, además, evidencia y verosimilitud. (1994, p. 21)

Lo mismo, ahora en palabras de Javier Blasco:

La literatura fantástica "reacciona" contra los límites en que los esquemas racionalistas pretenden encerrar la realidad, abriendo implícita o explícitamente, en el texto, la posibilidad de coexistencia de dos órdenes naturales o lógicos, aparentemente confrontados e incompatibles. (1994, p. 119)

Esta transgresión de la realidad real, ${ }^{2}$ característica de lo fantástico, se realiza a través de la aparición en el relato, del suceso fantástico, el cual se define como un evento fuera de la lógica que rige la realidad real del lector, pero que es inscrito por el autor en un escenario (previamente dibujado dentro del cuento) acorde con la lógica que rige esta realidad. No obstante, no solo se trata de una transgresión de aquella realidad que se refiere "a lo que existe y lo que no" en términos de percepción de los sentidos, etc., sino también, de una transgresión y transformación de la realidad social en la que vive el escritor de narraciones fantásticas. Así opina Ana María Barrenechea cuando afirma que las revoluciones literarias, como las propuestas por Cortázar en cuanto a forma, y por Eco en lo relativo a creación de nuevos lenguajes, "abren también al género otras posibilidades bajo el signo de lo social, siempre que lo fantástico sea una puesta en cuestión de un viejo orden que debe cambiar urgentemente" (citada por Dónoan, 1994, p. 131). De igual manera, Dónoan asegura que "el mundo de la ficción y especialmente su creación más imaginativa, surge de un centro interior, íntimo, que a su vez asume lo más profundo de la realidad social como comprensión o como crítica" (Dónoan, 1994, p. 131).

Barella et al. (1994) sostienen que luego de lo expuesto por Todorov (1998), Vax (1980), Jacques Finné (citado por Barella et al., 1994) y algunos otros, toda la crítica maneja el supuesto de que una de las características principales (rasgo específico) de la literatura fantástica es la "vacilación en el comportamiento del lector ante el acontecimiento extraño" (Barella et al., 1994, p. 12). Respecto a este hecho, Javier Gómez Montero considera que se trata de un evento que

\footnotetext{
2 Para Vargas Llosa (1971), la realidad real se sitúa fuera de la narración; esto es, la realidad en la que se mueve el escritor, con la que interactúa cotidianamente; definida no solo como los sucesos sociales y políticos que enmarcaron las narraciones fantásticas del período escogido, sino en conjunción con las ideas y paradigmas que gobiernan y guían dichos sucesos.

solo sucede a partir del Romanticismo, cuando se comienzaa manejar laidea de "los estados sicológicos que representa[n] la acción en los personajes o provoca[n] en el receptor (terror, inquietud, incertidumbre, etc.) ante el resquebrajamiento de las leyes que rigen la realidad empírica habitual" (Gómez Montero, 1994, p. 53). Esto demuestra, según H. P. Lovecraft (1984), la importancia y fuerza que tiene el miedo, constituyendo los relatos sobre el horror en los primeros relatos fantásticos; estos recogen del género del terror el juego con el miedo y, del género fantástico, el que el miedo sea hacia lo desconocido, desde donde proponen la transgresión característica de la realidad real.

Según Ángeles Ezama, otro elemento necesario para crear el "efecto fantástico" es el uso de la narración en primera persona, puesto que "con ella nos situamos en el centro mismo de la consciencia enajenada del personaje, sin intermediarios" (Ezama Gil, 1994, p. 80).

Como último punto en la definición de lo fantástico, resalto la concordancia en varios autores, quienes coinciden en denominar estas narraciones como "cuento fantástico", no "novela", asunto que, según J.C. Mena (1994), se explica por cuanto una de las principales características de la novela es su estrecha relación con la realidad, preocupándose por narrarla al más mínimo detalle, organizar (encuadrar) su argumento dentro y a partir de ella, sin presentar necesariamente una irrupción en su continuidad, o una transgresión en su orden habitual, como ya se ha dicho que es característico en el cuento fantástico.

En términos de argumento, Calvino asegura que el tema común del género fantástico es "la relación entre la realidad del mundo que conocemos y habitamos a través de la percepción, y la realidad del mundo del pensamiento que habita en nosotros y nos dirige" (Calvino, 1987, p. 9).

Por el contrario, Todorov (1998) opina que los temas de lo fantástico se dividen en dos grandes categorías:

Temas del "yo": En estos se trabaja la relación entre el hombre y su mundo, lo que lo rodea; se trata de la percepción o consciencia que este hombre tiene de su entorno; en una posición pasiva frente al mismo, de observador aislado. Son los temas de la "mirada".

Temas del "tú": Aquí la relación se establece entre el hombre y su propio "deseo" (relacionado también con la interacción con otros hombres). A diferencia del anterior, el papel del humano ahora es fuertemente activo, se plantea como un transformador. Son los temas del "discurso". 


\section{Literatura fantástica y contexto en el Cono Sur}

Sobre los autores de literatura fantástica, Leopoldo Lugones y Horacio Quiroga, cabe afirmar:

Al escoger una realidad sobre la cual escribir, no se deciden por una realidad de carácter social, sino por la denominada realidad real. Consideran que ésta siempre generará presión e inestabilidad en las mentes curiosas, ya que por más que se ahonde en ella siempre parece esconder misterios inexplicables ante la evidencia de fenómenos sobre los cuales nadie parece tener la respuesta idónea. (Bazán, 2003)

Por ello, aparentemente, no escogen temas políticos o sociales para denunciar o criticar la injusticia o desigualdad, más bien centran la crítica en otro orden, en la ciencia y su manera de explicar la realidad, en lo que existe y en lo que no:

Las preocupaciones sobre la realidad real, y no exclusivamente sobre la realidad social, son preocupaciones de orden intelectual y abarcan varios ámbitos, uno de ellos situado al nivel del lenguaje. Es precisamente ésta una de las temáticas más importantes dentro del movimiento modernista, la cual se refleja en varios de sus relatos (ver ejemplos en: La Estatua de Sal, e Yzur-Lugones - ${ }^{3}$ y Las Rayas —Quiroga-4). (Bazán, 2003)

La identidad nacional argentina, en mucho construida a partir de la figura del gaucho como imaginario, genera un terreno abonado para la escritura fantástica y su transgresión de la realidad real:

De esta manera, los inicios del género fantástico latinoamericano, tal como se manifiesta en los relatos de Horacio Quiroga y Leopoldo Lugones, son una mezcla de tres componentes: un ideal europeizante (evidente en el uso del lenguaje y los temas de mitología griega - sobre todo en Lugones-), el sistema de valores del gaucho y el entorno natural propio de zonas como Misiones, tan importante en las narraciones quiroguianas.

En este punto, pueden inscribirse los aportes críticos de Dussel (1999) y Mignolo (2000) consistentes en percibir detrás de intentos como los de los modernistas por buscar "lo propio" y ensalzar imaginarios como el del gaucho en Argentina, una manera de prolongar la colonialidad (Quijano, en: Mignolo, W. 2000) en las mentes, puesto que ver el mundo desde esta perspectiva, es verlo a la manera europea. (Bazán, 2003).

\footnotetext{
${ }^{3}$ Lugones, Leopoldo (1985a).

${ }^{4}$ Quiroga, Horacio (1977).
}

Pero para Lugones y Quiroga existen unas "fuerzas extrañas" de la naturaleza que influyen sobre el hombre o sobre la búsqueda de "lo humano" que permitirían pensar al hombre latinoamericano por fuera de la "racionalidad instrumental" europea, aunque esta última sea real:

En las narraciones sobre la selva y lo pseudo-científico (Sarlo, 1992) encuentran una manera no eurocéntrica de pensar su entorno, de evidenciarlo desde él mismo, tal como se presenta a sus sentidos. ...Un espacio como la selva de Misiones, con la "fuerza" que ésta posee, no tiene equivalente en Europa, y por lo tanto no existe para ésta.

Conviene destacar que dicha situación en los dos literatos no se da de manera consciente, es decir, no están tratando de forjar una teoría al respecto, lo cual se evidencia en la doble dinámica de realizar ensayos y disertaciones que hablen sobre Latinoamérica a la manera eurocéntrica (como es el ejemplo en algunos ensayos que hacen referencia a la situación argentina en Lugones), y al mismo tiempo proponer en sus relatos la existencia de otra realidad que perciben (aspecto que le da el calificativo de real, existente), pero que no pueden explicar con las herramientas de conocimiento que poseen. Tal dinámica, en un nivel literario, sólo podría lograrse desde los relatos fantásticos, debido a su característica definitoria de juego y transgresión de la realidad real. ${ }^{5}$

Es necesario aclarar la manera como esta literatura propone transgredir el orden de la realidad real del lector, lo cual sucede a partir de la adecuación de una atmósfera dentro del cuento que siga los preceptos de la realidad real, esto es, lo más parecido posible a la realidad en la que el lector se mueve (es preciso que él identifique su mundo y las leyes que lo rigen); luego de esto, sucede un hecho fantástico que controvierte la lógica expuesta con anterioridad, pero siempre constituido con los mismos materiales que el ambiente 'ordinario' en el cual se enmarca. Se requiere, además, en este orden narrativo que no quede clara (para el lector) la proveniencia del suceso fantástico, es decir, que no se determine si la explicación dada al suceso es sobrenatural o racional, de manera que genere en el lector una vacilación ${ }^{6}$ entre ambos tipos de explicación (Bazán, 2003).

\footnotetext{
${ }^{5}$ Este concepto es desarrollado por Blasco, Javier (1994),y Dónoan (1994).
} ${ }^{6}$ Concepto desarrollado por Todorov (1998). 
que consideran fundamentales para cada sector social en particular, y a partir de los cuales proponen entender la forma de pensar de toda una sociedad.

$\mathrm{Su}$ propuesta es, entonces, pasar "del texto al contexto, regresando de nuevo a éste hasta lograr encontrar una ruta en un mundo mental extraño" (Darnton y Valdés, 1987, p. 13). Es la búsqueda de lo que ellos llaman el "sentido común del mundo real", que determina el ordenamiento mental de una sociedad y, por vía suya, el social. Esto sería lo que entronca con nuestra mencionada relación contextoliteratura fantástica, en adelante contexto o entorno sociocultural.

De igual manera, Rolando Mellafe (1986) sugiere, para un estudio cualquiera sobre la personalidad de un pueblo, no tener en cuenta solo los acontecimientos faustos, felices y gloriosos de su historia, sino también el "acontecer infausto" de un pueblo (las crisis y los momentos coyunturales); asegura que el "acontecer infausto tiraniza” el diálogo entre la psiquis humana y la naturaleza, y estas "tensiones que el reiterado encuentro (diálogo) produce, el terror que muchas veces nos inspira, provoca fenómenos colectivos que se traducen en modos de ser y de actuar" (Mellafe, 1986, p. 287). Esto significa cubrir otro pedazo de la historia de un pueblo que no siempre es bien vista por los historiadores, pero que, como dice Mellafe, cumple un papel fundamental en el proceso de construcción del carácter y la identidad nacional de un pueblo.

Por otro lado, dentro de la disciplina de la historia, hay un campo que se acerca mucho a lo planteado aquí, y es la Historia de las Mentalidades, la cual nace en los años 60, cuando algunos teóricos pertenecientes a l'école sociologique, como Philippe Ariès, Fernand Braudel, George Duby y Michel au Bobbel, se preguntan por una metodología que permita - desde la historia- estudiar las actitudes, los valores y los sentimientos colectivos, en sus transformaciones a través del tiempo. La Historia de las Mentalidades (que sale de la escuela de las mentalidades) es analizada sobre períodos largos de tiempo, por cuanto se entiende que las mentalidades necesitan de mucho tiempo para cambiar o transformarse (Ana Luz Rodríguez, comunicación personal).

La Historia de la Cultura -otro campo como la historia de las mentalidades- trabaja sobre la interpretación de textos, de los cuales, en palabras de Mellafe, se "estudia las expresiones resultantes de vivencias que individuos o grupos tienen de la acumulación de ciertas experiencias básicas" (1986, p. 280), tales como "nacer, morir, enfermar, comer, sentir angustia, amor, alegría, etc."
Para el tipo de análisis a realizar en esta investigación, sobre una producción cultural como lo es la literatura, el tipo de historias nacionales que más ayudan a interpretar las costumbres, comportamientos, ideales, creencias, etc., de un pueblo (lo definido en este trabajo como contexto sociocultural), es el tipo de historias como la de Mellafe (1986), historias de las mentalidades. La Historia social de Chile y América es relevante en este momento, puesto que realiza un estudio acerca de la historia latinoamericana durante la colonia, para terminar usando los datos conseguidos en una historia de la mentalidad (carácter) chilena; intentando descubrir las "experiencias vitales" (mencionadas arriba), para luego examinarlas y entender cómo evolucionaron y en qué medida están actuando en las distintas etapas de la historia chilena. Se trata de un enfoque útil para nosotros acerca del análisis del "acontecer infausto":

$\mathrm{Al}$ respecto, es necesario ampliar la concepción sobre el hombre que Quiroga y Lugones reflejan en los personajes de las narraciones fantásticas; se trata en su mayoría de hombres condenados al "fracaso" desde la perspectiva de occidente, aunque no en la de los personajes. Lo anterior se evidencia sobre todo en las narraciones "pseudo-científicas", en las cuales se conciben máquinas fantásticas con posibilidades increíbles, pero donde también (en todos los cuentos leídos sobre este tema) el proyecto culmina en un fracaso rotundo que pone en ridículo a los inventores-protagonistas dentro del cuento; sus experimentos carecen del rigor científico y seriedad en la experimentación esperable para empresa tal; sus gestores son hombres borrachos o seres con arranques de locura, en fin, todo lo contrario del prototipo de hombre de ciencia responsable por el avance y futuro de la humanidad, según lo postula el ideal positivista. Es como si "lo importante" no estuviese en el carácter productivo, o en los resultados de su tarea, sino tal vez en lo que se va descubriendo por el camino, y en el placer que produce realizarla. (Bazán, 2003)

Otro campo afín a nuestro tema, ahora dentro de la sociología, es la Sociología Literaria, la cual a pesar de que en la mayor parte de textos encontrados se preocupan más por realizar crítica literaria, e incluso Historia de las Mentalidades, que por un análisis social de la obras literarias, como se esperaría, es de gran utilidad, por cuanto el enfoque sociológico en una Historia de las Mentalidades, como es el caso del libro de Mellafe (1986), no solo es interesante, sino relevante a un análisis como el aquí propuesto.

En el artículo escrito por Jacques Leenhardt (1971) se proponen dos campos de acción para la sociología literaria:

1) La literatura como producto de consumo (dentro de una sociedad de consumo literario).

2) La literatura como parte integrante de la realidad social (en una sociedad que es sujeto de creación literaria). 
En este estudio es útil la Sociología Literaria, siempre que entienda la sociedad como un "sujeto" creador de ciertos "productos culturales" $y$, sobre todo, sea consciente de que la literatura se inscribe dentro de un entorno social al cual determina, pero por el cual es, al mismo tiempo, determinada. Leenhardt (1971) comenta acerca de la concepción que sobre el segundo campo de acción de la Sociología Literaria (mencionado arriba) tiene Auerbach:

Las transformaciones de la realidad social y las de las maneras de pensar y de sentir a las que aquellas sirven de vehículo repercuten no sólo en el contenido de las obras literarias, sino también en su estructura formal, en su estilo y hasta en su estructura sintáctica. (Citado por Leenhardt, 1971)

Por otra parte, llama la atención que en el artículo de G.N. Pospelov, "Literatura y sociología”, este asegure que solo la literatura "puede abarcar [...] toda la multiplicidad de las experiencias sociohistóricas vividas por la humanidad" (Pospelov, 1971, p. 75), lo cual valida el presente proyecto.

Indica más adelante este autor que el análisis se centra en la búsqueda por "la ideología de una comunidad", lo que comprende convicciones intelectuales, síntesis de las ideas que sobre la vida manejan los hombres y los sentimientos y aspiraciones que originan dichas convicciones; a todo este primer grado de la "conciencia ideológica" se le llama "visión de mundo". Así, cabe anexar a la presente investigación, además de la búsqueda del entorno social en el que se produce la literatura de nuestro interés, las visiones de mundo que comandan a los productores de dicha literatura, a las que igualmente se podrían sumar las "prácticas ideológicas" propuestas por Pérus (1982), quien considera que la Sociología Literaria se ha encargado tradicionalmente de buscar la "función social" de la literatura (función concebida como transformable). Sin embargo, en su estudio pretende además (entendiendo la literatura como una "práctica ideológica concreta") dilucidar sus múltiples "niveles de determinación"; esto es, entender el "funcionamiento concreto del hecho literario y del texto en su relación a la vez con la escritura y la lectura" (Pérus, 1982, p. 15), al igual que lo propuesto por Leenhardt (1971):
Las narraciones fantásticas de Quiroga y Lugones transgreden también la forma de escribir literatura fantástica, así como los temas clásicos usados por ella (lo que más adelante con Borges y Cortázar alcanza su clímax). Prueba de ello es la ausencia en varios de los cuentos de un tema como el terror y de una técnica como el suspenso, ingredientes fundamentales para la mayoría de cuentos fantásticos clásicos; o al proponer, además de los relatos en los que se aborda el tema de las pasiones humanas llevadas al límite (que en los clásicos es muy común, junto con el tema de las apariciones fantasmagóricas), otros de carácter más filosófico que emocional, abanderando reflexiones sobre el entorno natural, o la relación hombre-animal (entre otros). (Bazán, 2003)

En este mismo sentido, Pérus (1982) realiza un análisis acerca de la relación entre mito y realidad en Don Segundo Sombra. Sostiene que, mientras para muchos críticos (incluido el propio escritor) esta obra es una "copia" de la realidad del momento en que es concebida y describe lo que es "ser gaucho", "ser argentino", mostrando al gaucho como "la esencia del carácter nacional", la novela es más bien un intento por conciliar, por medio de la ficción literaria ("ilusión concreta"), "el mito gaucho con la apropiación privada del suelo pampeano" (Pérus, 1982, p. 253). Esta conciliación se presenta al servicio de la oligarquía estanciera (desde donde es gestada la novela), como una forma de presentar su historia personal y de legitimar su dominación. Para Pérus, Ricardo Güiraldes no hace uso de la realidad en la que el gaucho se encuentra en el momento de escribir la novela, sino más bien de la realidad del mito que sobre el gaucho se ha difundido, aseverando que esto demuestra que la literatura no atiende (en todos los casos) a una necesidad de realidad, como a una "necesidad ideológica de clase". 


\section{Referencias bibliográficas}

Alazraki, J. (1983).En busca del unicornio: los cuentos de Julio Cortázar. Elementos para una poética de lo neo-fantástico. Madrid: Editorial Gredos S. A.

Barella, J., Guerri, M. J. C., y Carbajo, F. G. (1994). Madrid en la novela. Madrid: Comunidad de Madrid.

Bazán, N. (2002). ¿No es real lo fantástico? La literatura fantástica en el Cono Sur en relación con su contexto social y cultural (Trabajo de grado). Departamento de Antropología, Universidad de los Andes, Bogotá.

Bazán, N. (2003) Literatura fantástica: Una nueva lógica que busca expresar y entender el cono sur. Revista Grafía, 1, pp. 137-149.

Belevan, H. (1976). Teoría de lo fantástico: apuntes para una dinámica de la literatura de expresión fantástica. Madrid: Anagrama.

Bioy Casares, A. (1967). Prólogo. En J. L. Borges, S. Ocampo y A. Bioy Casares, Antología de la literatura fantástica. Buenos Aires: Sudamericana.

Blasco, J. (Marzo-abril, 1994) Extraordinario, pero no fantástico. El género de las misceláneas renacentistas. ANTHROPOS, 154 (155).

Borges, J. L. (1965). Leopoldo Lugones. Buenos Aires: Editorial Pleamar.

Borges J. L. (1998). Ficciones. Argentina: Emecé Editores.

Calvino, I. (1987). Cuentos fantásticos del XIX [Volumen primero y segundo]. Madrid: Ediciones Siruela.

Carmagnani, M. (1984). Estado y sociedad en América Latina 1850-1930. Barcelona: Editorial crítica.

Darnton, R., y Valdés, C. (1987). La gran matanza de gatos y otros episodios en la historia de la cultura francesa. México: Fondo de Cultura Económica.

Dónoan (Marzo-abril, 1994). Las invenciones de la literatura fantástica abren nuevas dimensiones críticas y alternativas a la verificación histórica del presente. ANTHROPOS, 154 (155).

Dussel, E. (1999). Más allá del eurocentrismo: el sistema mundo y los límites de la modernidad.En S. Castro Gómez, O. Guardiola Rivera y C. Millán Benavides (eds.), Pensar (en) los intersticios. Bogotá: Colección Pensar, Pontificia Universidad Javeriana.

Ezama Gil, M. D. L. A. (1994). Cuentos de locos y literatura fantástica: Aproximación a su historia entre 1868 y 1910. ANTHROPOS, (154), pp. 77-82.

Gutiérrez, F. (1994) El universo fantástico de Alfahuí. Anthopos (154), pp.64-68

Gil, P.V. (1994). Lo fantástico como categoría estética. ANTHROPOS, (154), pp. 21-24.
Gómez Montero, J. (1994). lo fantástico y sus límites en los géneros literarios durante el siglo XVI. ANTHROPOS, 154 (155).

Goldmann, L., Leenhardt, J., Pospelov, G. N., Eco, U., Lukács, G., Mouillaud, G., Waltz, M. (1971). Sociología de la creación literaria. Buenos Aires: Nueva Visión.

Hale, C. A. (1991). Ideas políticas y sociales en América Latina, 1880-1930»; En: Leslie Bethell, (ed.) «Historia de América Latina» Tomo VIII. Barcelona: Editorial crítica

Leenhardt, J. (1971). La sociología de la literatura: algunas etapas de su historia. Sociología de la creación literaria, 45-71

Lovecraft, H. P. (1984). HP Lovecraft. Paris: Éditions de l'Herne.

Lugones, L. (1985a). La estatua de sal. Madrid: Ediciones Siruela.

Lugones, L. (1985).Un fenómeno inexplicable e Yzur.Madrid: Ediciones Siruela.

Mellafe, R. (1986). Historia social de Chile y América. Chile: Editorial Universitaria.

Mena, J. C. (1994). Fantasías. ANTHROPOS, 154(155), pp. 6164.

Mignolo, W. (2000).Diferencia colonial y razón postoccidental. En S. Castro Gómez (ed.), La reestructuración de las ciencias sociales en América Latina. Bogotá: Colección Pensar, Pontificia Universidad Javeriana.

Pérus, F. (1982). Historia y crítica literaria: el realismo social y la crisis de la dominación oligárquica. Cuba: Casa de las Américas.

Pospelov, G. N. (1971). Literatura y sociología. En L. Goldmann, J. Leenhardt, G. N. Pospelov, U. Eco, G. Lukács, G. Mouillaud, M. Waltz, Sociología de la creación literaria. Buenos Aires: Nueva Visión.

Quiroga, H. (1937). Cuentos [tomo III]. Montevideo: Claudio García y Cía. Editores.

Quiroga, H. (1977). Anaconda. Buenos Aires: Editorial Losada.

Sarlo, B. (1992). La imaginación técnica. Sueños modernos de la cultura argentina. Buenos Aires: Ediciones Nueva Visión.

Todorov, T. (1998). Introducción a la literatura fantástica. México: Ediciones Coyoacán.

Todorov, T., Barthes, R., et al. (1970). Lo verosímil. Buenos Aires: Editorial Tiempo Contemporáneo.

Vargas Llosa, M. (1971). García Márquez: Historia de un deicidio. Barcelona-Carácas: Monte Ávila Editores.

Vax,L. (1980). Obras maestras de la literatura fantástica. Madrid: Taurus.

Zum Felde, A. (1959). Indice crítico de la literatura hispanoamericana; La Narrativa [Tomo II]. México: Editorial Guaranía.

Nadia Juliana Bazán: Clarinetista. Antropóloga con opción en teoría musical de la Universidad de los Andes de Colombia. Posgrado en Derechos Humanos de Columbia University, New York. Actualmente se desempeña como Coordinadora de apoyos en Fondo de Acción Urgente, FAU.

nadiabaz@gmail.co

Artículo recibido en marzo de 2013 y aceptado en septiembre de 2013 